Research Article

\title{
Deep Learning-Based CT Images in Pulmonary Function Assessment of Patients Who Underwent Laparoscopic Surgery under Guidance of Electrical Impedance Tomography
}

\author{
Chen Zhu $\mathbb{D},,^{1,2}$ Bin Wang $\left(\mathbb{D},{ }^{3}\right.$ Lei $L i \mathbb{D}^{3},{ }^{3}$ and Tianzuo Li $\mathbb{D}^{1}$ \\ ${ }^{1}$ Department of Anesthesiology, Beijing Shijitan Hospital, Capital Medical University, Beijing 100038, China \\ ${ }^{2}$ Department of Anesthesiology, Beijing Friendship Hospital, Capital Medical University, Beijing 100050, China \\ ${ }^{3}$ Department of Anesthesiology, Emergency General Hospital, Beijing 100028, China
}

Correspondence should be addressed to Tianzuo Li; 150511130@stu.sxit.edu.cn

Received 22 June 2021; Revised 12 August 2021; Accepted 14 August 2021; Published 6 October 2021

Academic Editor: Gustavo Ramirez

Copyright (c) 2021 Chen Zhu et al. This is an open access article distributed under the Creative Commons Attribution License, which permits unrestricted use, distribution, and reproduction in any medium, provided the original work is properly cited.

This research aimed to study the application of CT images based on deep learning in pulmonary function assessment of patients who underwent laparoscopic surgery under the guidance of electrical impedance tomography (EIT). Sixty patients undergoing laparoscopic surgery were taken as the research subjects, who were randomly labelled as control group and experimental group. Based on deep learning, the empty convolution-combined fully convolutional neural network optimization algorithm (ECFCNN) was proposed, which was adopted to evaluate the pulmonary function of 60 patients and was compared with convolutional neural network $(\mathrm{CNN})$ algorithm. The clarity of the edge contour of the image segmented by ECFCNN was better than that segmented by $\mathrm{CNN}$. Average arterial pressure (MAP) and heart rate (HR) were recorded before induction (T1), 10 min before pneumoperitoneum (T2), $10 \mathrm{~min}$ after pneumoperitoneum (T3), $10 \mathrm{~min}$ before extubation (T4), and $10 \mathrm{~min}$ after extubation (T5), respectively. Oxygenation index $\left(\mathrm{PaO}_{2} / \mathrm{FiO}_{2}\right)$, alveolary-arterial partial pressure of oxygen (A-ADO2), and respiratory index (RI) were recorded. The sharpness of the segmentation image edge contour of the algorithm model in this study was higher than that of the convolutional neural network. Compared with T1, T2-T4 MAP in 2 groups was decreased $(P<0.05)$. Compared with T1, T2T5 HR was significantly decreased $(P<0.05)$. Compared with $\mathrm{T} 2, \mathrm{~T} 5 \mathrm{PaO}_{2} / \mathrm{FiO}_{2}$ in control group was significantly decreased $(P<0.05)$. Compared with the control group, T5 $\mathrm{A}-\mathrm{aDO}_{2}$ was decreased $(P<0.05)$. To sum up, EIT-guided lung protective ventilation can assess the pulmonary function of patients who underwent laparoscopic surgery, reduce the incidence of atelectasis, and improve postoperative lung oxygenation.

\section{Introduction}

In recent years, with the continuous progress of surgical operations, the requirements for the size of the wound, the degree of pain, and the prognosis of the operation have become higher and higher. Laparoscopic surgery has the advantages of small wounds, low blood loss, short recovery time, and small recovery scars. At present, it is widely utilized clinically [1].

EIT is a medical technology that takes the impedance distribution of human tissues and organs as the imaging target. It reconstructs the internal change distribution image of the human body by applying safe electrical excitation to the human body and measuring the response voltage signal outside the body [2]. Under the guidance of EIT, the pulmonary function of patients who underwent laparoscopic surgery can be assessed. The clinical routine examination items are mainly lung volume measurement, pulmonary ventilation function measurement, and arterial blood gas analysis [3]. Whether it is a patient with healthy pulmonary function or a patient with impaired pulmonary function, EIT can clearly image the blood flow of the lungs, and the results match with CT scans [4]. CT scans a certain part of the human body using X-rays and a highly sensitive detector, which has fast scanning time and clear images [5]. In recent years, CT images have been widely adopted in the field of 
medical clinical diagnosis. Tomography scans of the injured parts of the human body can help determine the disease status by analyzing the images. CT examination has become an indispensable way to assess pulmonary function in the medical field [6]. Although there are still many pulmonary function assessment methods under the guidance of EIT in the past, there is no relevant literature on adopting CT imaging combined with EIT in pulmonary function assessment of patients who underwent laparoscopic surgery [7]. Medical image segmentation algorithms mainly include three types: traditional segmentation, mechanical segmentation, and deep learning segmentation. Deep learning is widely utilized in computer vision and sound effects. It can organize large amounts of data in an orderly manner, extract image features, and efficiently deal with intricate problems [8].

Based on deep learning, the ECFCNN algorithm was proposed, which was compared with $\mathrm{CNN}$ algorithm and applied to the evaluation of pulmonary function of 60 patients undergoing laparoscopic surgery. It aimed to explore the diagnosis and prognosis of pulmonary function in patients undergoing laparoscopic surgery under the guidance of EIT.

\section{Materials and Methods}

2.1. Basic Information. From October 2017 to December 2019,60 patients who were medically diagnosed with urinary system diseases and required laparoscopic surgery in hospital were taken as the research objects. Among them, 21 were males and 39 were females, with an average age of $59.23 \pm 11.32$ years. The random number table method was adopted to classify patients into two groups. The control group received normal ventilation while experimental group received lung protective ventilation, with 30 cases in each. The study had been approved by the Medical Ethics Committee of the Hospital, and the patients and their families understood the situation of the study and signed informed consents, respectively.

Inclusion criteria were as follows: (I) patients diagnosed with urinary system diseases and undergoing laparoscopic surgery; (II) patients with complete clinical data and imaging data; (III) patients with grades I-II according to the American Association of Anesthesiologists (ASA) grading.

Exclusion criteria were as follows: (I) patients with cardiovascular disease, mental illness, speech impairment, or hearing impairment; (II) patients with severe spinal deformity; (III) patients with breathing difficulties, asthma, or smoking; (IV) patients with unclear CT images.

2.2. Anesthesia and Monitoring of Laparoscopic Surgery. The patients did not take medicine before entering the laboratory. After the patient entered the operating room, a peripheral venous channel was established. Inhalation of oxygen was through a mask, and the American Aspect 2000 was adopted to monitor the bispectral index (BIS) of the brain electricity. The German Philips MP60 monitor was utilized to monitor and record the patient's MAP, HR,
$\mathrm{A}-\mathrm{aDO}$, and EIT. The patient was anesthetized after 10 minutes of oxygen inhalation and intravenously injected with midazolam $0.045 \mathrm{mg} / \mathrm{kg}$, propofol $1.5 \mathrm{mg} / \mathrm{kg}$, sufentanil $0.5 \mu \mathrm{g} / \mathrm{kg}$, and rocuronium $0.9 \mathrm{mg} / \mathrm{kg}$. When the patient's BIS was less than 602, the Datex-Ohmeda avance anesthesia ventilator from Datex-Ohmeda in the United States was utilized for tracheal intubation. The experimental group was ventilated with low tidal volume at $7 \mathrm{~mL} / \mathrm{kg}$, while control group was ventilated with conventional tidal volume at $9 \mathrm{~mL} / \mathrm{kg}$ (the ideal weight: male $(\mathrm{kg})=50+0.91 \times$ (height152.4); female $(\mathrm{kg})=45.5+0.91 \times($ height-152.4)). The frequency of respiration was adjusted according to the carbon dioxide concentration at the end of the respiration, and the inhalation-expiration ratio was set to $1: 2$. Anesthesia was maintained by intravenous injection of propofol $6 \mathrm{~mL} / \mathrm{kg} / \mathrm{h}$ and remifentanil $0.15 \mu \mathrm{g} / \mathrm{kg} / \mathrm{min}$. The fluctuating pressure was set to $13 \mathrm{mmHg}$. After the pneumoperitoneum started, we needed to constantly adjust the breathing parameters. Adjustment should be made at any time according to the changes of MAP and HR. For hypotension, MAP was less than $60 \mathrm{mmHg}$, lasting more than $40 \mathrm{~s}$, with intravenous injection of phenylephrine $30 \mu \mathrm{g}$. For hypertension, MAP was greater than $85 \mathrm{mmHg}$, lasting longer than $40 \mathrm{~s}$, with intravenous nicardipine $0.3 \mathrm{mg}$. If $\mathrm{HR}$ was less than 40 beats $/ \mathrm{min}$, intravenous atropine was $0.30 \mathrm{mg}$. If the $\mathrm{HR}$ exceeded 85 beats $/ \mathrm{min}$, esmolol $0.45 \mathrm{mg} / \mathrm{kg}$ was injected intravenously.

\subsection{Observation Indexes for Patients Undergoing Laparoscopic} Surgery. Before the operation, statistics of the patient's gender, age, weight, and other general information were implemented. Before induction (T1), 10 minutes before pneumoperitoneum (T2), 10 minutes after pneumoperitoneum (T3), 10 minutes before extubation (T4), and 10 minutes after extubation (T5), the patient's MAP and HR were recorded. The oxygenation index $\left(\mathrm{PaO}_{2} / \mathrm{FiO}_{2}\right)$, $\mathrm{A}-\mathrm{aDO}_{2}$, and respiratory index (RI) were analyzed and recorded using the GEM Premier 3500 blood gas analyzer from Wefen, USA. The operation time, anesthesia time, pneumoperitoneum time, and the number of patients with postoperative adverse events such as hypercapnia, agitation, and hypoxemia were recorded.

2.4. CT Scan for Patients Undergoing Laparoscopic Surgery. GE light speed VCT 64-slice spiral CT scanner was adopted, and patients shall remove metal jewelry on the chest before scanning. The iodine allergy was tested in advance, and the scanning process was explained to keep the patient calm. First, left and right plain scan on the chest was performed, followed by top-down scan. The scan range was from the upper thorax to the diaphragm, and then an enhanced scan was taken. The scanning layer thickness was $0.625 \mathrm{~mm}$, the scanning interval was $0.5 \mathrm{~mm}$, the pitch was $0.984: 1$, the matrix was $512 \times 512$, the voltage was $120 \mathrm{kV}$, and the current was $220-500 \mathrm{~mA}$. The bolus injection method was adopted to inject the contrast agent. First, $15 \mathrm{~mL}$ of normal saline was injected from the vein to check for subcutaneous leakage. The injection rate of contrast agent iopromide was $3.5 \mathrm{~mL} / \mathrm{s}$, 
and the injection volume was $80 \mathrm{~mL}$. Then, in the same way, $35 \mathrm{~mL}$ of saline was injected to reduce the concentration of the contrast agent in the superior vena cava. After the contrast medium injection, when its concentration reached the threshold, the scan would be performed with a delay of 5 s.

2.5. ECFCNN Algorithm. Neuron is the basic functional unit of neural network structure, as illustrated in Figure 1. In Figure 1, X1, X2, and X3 represent different input values; V1, $\mathrm{V} 2$, and $\mathrm{Vm}$ represent different weights; $\sum \mathrm{XiVi}+\beta$ represents the sum of the product of input and weight and the parameter $\beta$, and $g(\cdot)$ represents a nonlinear function. A large number of neurons together form a neural network structure. Based on deep learning FCNN and empty convolution, ECFCNN algorithm was proposed for lung CT image segmentation.

The internal structure of ECFCNN model mainly includes convolutional layer and empty convolution. The essence of convolution operation is integral operation, and the calculation is as follows:

$$
(g \cdot h)(s) \triangleq \int_{-\infty}^{\infty} g(\gamma) h(s-\gamma) \mathrm{d} \gamma .
$$

In (1), function $g$ and function $h$ represent continuous functions, and both are integrable functions in the real number range, $S$ represents the convolution kernel, and $r$ represents the number of network layers. Assuming that the input two-dimensional vector $P$ is $6 \times 6$ and the convolution kernel $Q$ is $4 \times 4$, then, the output $L$ is a $4 \times 4$ two-dimensional tensor. The equation is as follows:

$$
L(i, j)=(P \cdot Q)(i, j)=\sum_{a} \sum_{b} P(a+i, b+i) \cdot Q(a, b) .
$$

According to (2), the size of the input image ( $i \times i)$ directly affects the size of the output image $L \times L$, and it will also be affected by the size of the convolution kernel $(q \times q)$ during the operation. To better segment the edges of the image, supplementary parameter $o$ is often added. The specific equation is as follows:

$$
l=\left\lfloor\frac{i-q+2 o}{t}\right\rfloor+1 .
$$

Model networks often have multilayer superimposed hollow convolution, and using zigzag structure can avoid the superposition of information and maintain the continuity of information. The zigzag structure uses time intervals of different lengths to integrate information from different distances. The equation is as follows:

$$
N_{i}=\max \left[N_{i+1}-2 \lambda_{i}, N_{i+1}-2\left(N_{i+1}-\lambda_{i}\right), \lambda_{i}\right] .
$$

In (4), $\lambda_{i}$ refers to the hole interval of the $i$ th layer and $N_{i}$ is the hole interval of the last layer and the maximum value of the hole interval. In this work, the void interval used is (2, $4,5)$. The essence of the operation of the convolutional layer is to perform weighted summation. The function used in this study is the Sigmoid function when classification output is conducted, and the Rlu function is used when image features

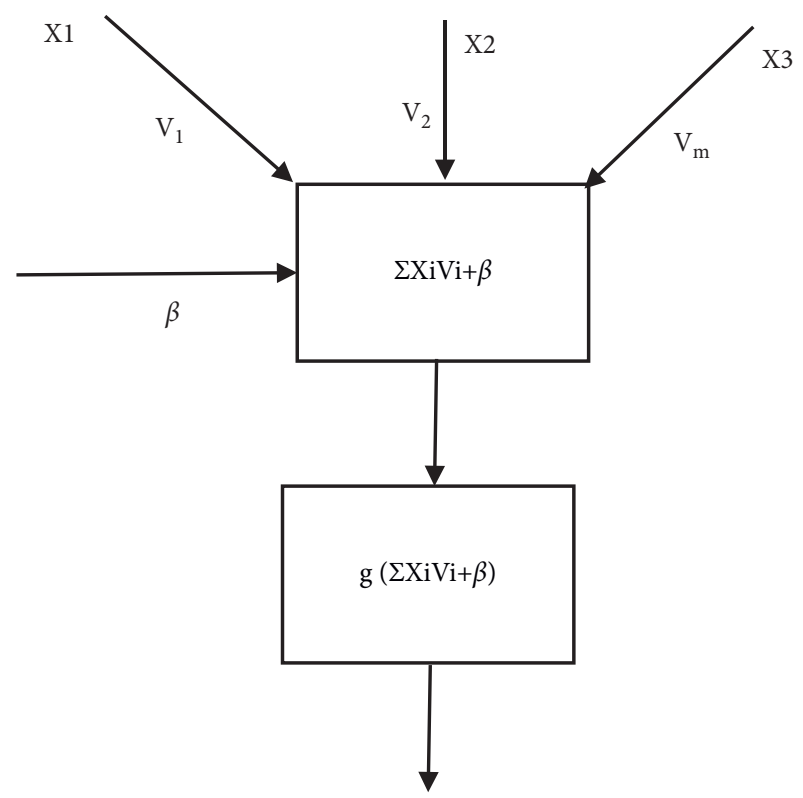

FIGURE 1: The basic structure of neurons.

are extracted. Among them, the calculation equation and derivative equation of the Sigmoid function are as follows:

$$
\begin{aligned}
\operatorname{Sig} & =\frac{1}{1+e^{-c}}=\frac{1}{1+e^{-(v x+b)},} \\
\operatorname{Sig}^{\prime}(c) & =\frac{e^{-c}}{\left(1+e^{-c}\right)^{2}} .
\end{aligned}
$$

The function values in (5) and (6) are all in interval $(0,1)$, and the Sigmoid function is a derivative function. The Rlu function can reduce the problem of the disappearance of the gradient, whose mathematical operation equation and derivative equation are as follows:

$$
\begin{aligned}
\operatorname{Rlu}(c) & =\max (0, c), \\
\operatorname{Rlu}^{\prime}(c) & = \begin{cases}0, & c<0 \\
1, & c>0\end{cases}
\end{aligned}
$$

In (7) and (8), when $c<0$, the derivatives of the Rlu function are all negative values, and the gradient is in a saturated state. When $c>0$, the derivatives of the Rlu function are all 1 , and the gradient will not disappear. Compared with the Sigmoid function, the calculation speed and convergence speed of the Rlu function are faster. For the input $P$, the degree of deviation of the gap between the model output $g(P)$ and the actual value $K$ is usually expressed via the loss function, which is recorded as $R(K, g(P))$, and the specific calculation equation is as follows:

$$
R=\frac{1}{2 m} \sum(K-g(P))^{2} .
$$

2.6. Statistical Methods. SPSS 22.0 statistical software was adopted for data analysis. Data meeting the normal distribution were expressed as mean \pm standard deviation, including general statistics of the two groups of patients, such 
as operation time, anesthesia time, pneumoperitoneum time, MAP, HR, A-aDO 2 , ROI, oxygenation index, and RI. Nonnormally distributed data were expressed in frequency and percentage (\%), including the loss of the two algorithms, the Dice coefficient, and the probability of adverse events. $T$ test and $\chi^{2}$ test were used for measurement data and enumeration data, respectively. The difference was statistically significant with $P<0.05$.

\section{Results}

3.1. Analysis of the Simulation Effect of Two Algorithms. Based on deep learning, ECFCNN algorithm was proposed, which was compared with $\mathrm{CNN}$ algorithm and applied to the evaluation of pulmonary function of 60 patients undergoing laparoscopic surgery. The loss function and Dice coefficient of the ECFCNN model and the CNN model were compared in Figure 2. The loss of the CNN model was 0.0523 and the Dice coefficient was 0.9635, while the loss of the ECFCNN model was 0.0469 and the Dice coefficient was 0.9712 . The loss function and Dice coefficient of ECFCNN model were obviously better than those of $\mathrm{CNN}$ model. The segmented images of the ECFCNN model can describe the overall outline of the lung. Moreover, the definition of the edge outline was higher than that of the CNN model.

\subsection{General Statistics of Patients Undergoing Laparoscopic} Surgery. The two groups of patients undergoing laparoscopic surgery were compared in terms of gender, age, and weight, and no considerable difference was found $(P>0.05)$. The operation time, anesthesia time, and pneumoperitoneum time were also compared; similarly, the difference was not considerable $(P>0.05)$, as illustrated in Figure 3. Therefore, there was no evident difference in the general information of the two groups of patients, and comparative experiments can be carried out.

\subsection{CT Imaging Characteristics of Patients Undergoing} Laparoscopic Surgery. The adrenal adenoma mass was about $4 \mathrm{~cm}$ in diameter with uneven internal density, which was considered to be caused by liquefactive necrosis. The medial side was close to the abdominal aorta, but there was still space, and the tumor was tightly connected to the vessels of the left renal pedicle (Figures 4(a) and 4(b)). Massive hepatocellular carcinoma was shown in the right liver, with hepatitis B cirrhosis. The preoperative abdominal enhanced CT indicated right liver occupation, which was very possibly to be liver cancer, and involvement of the right posterior portal vein was observed. After calculation of residual liver volume via $\mathrm{CT}$, it was found that the left liver volume of the patient was too small, and the residual liver volume only accounted for $25 \%$ of the total liver volume. The surgeon performed laparoscopic ligation of the right portal vein, liver splitting, and cholecystectomy under general anesthesia (Figures 4(c) and 4(d)).
3.4. Vital Signs of Two Groups of Patients Undergoing Laparoscopic Surgery. The average arterial pressure and HR of the two groups of patients at each time point were compared. It was found that, relative to that at T1, the MAP of control group and experimental group at T2-T4 time points were substantially reduced, and the difference was highly notable $(P<0.05)$, and that at T5 rebounded, but the difference was not considerable enough $(P>0.05)$ (Figure 5(a)). In contrast to that at T1, the HR at T2-T5 time points decreased obviously, with evident difference, as illustrated in Figure 5(b).

3.5. EIT Monitoring Data of Two Groups of Patients Undergoing Laparoscopic Surgery. Relative to that at T1, the lung area of interest in T5 increased after the pneumoperitoneum $(P<0.05)$. Figure 6 showed that, compared with T3, the lung area of interest in $\mathrm{T} 5$ increased after the pneumoperitoneum $(P<0.05)$, indicating that low tidal volume ventilation and positive end-expiratory pressure can remarkably improve the ventilation of the lung area.

3.6. Contrast of Pulmonary Function Indexes of Two Groups of Patients Undergoing Laparoscopic Surgery. The pulmonary function indexes, such as oxygenation index, $\mathrm{A}-\mathrm{aDO}_{2}$, and $\mathrm{RI}$, were compared between the two groups of patients. In contrast to T2 time point, the $\mathrm{PaO}_{2} / \mathrm{FiO}_{2}$ of control group at T5 decreased very notably, and the difference was substantial $(P<0.05)$. Figure 7(a) illustrated the notion that, relative to control group, the $\mathrm{PaO}_{2} / \mathrm{FiO}_{2}$ at T5 in experimental group was extremely superior to control group, with highly considerable differences $(P<0.05)$. A-aDO 2 at $\mathrm{T} 5$ was obviously reduced in experimental group, and the difference was highly notable versus control group $(P<0.05)$ (Figure $7(b))$. Compared with that at T2, the RI at T3 and T5 in experimental group was evidently reduced. RI at T5 in experimental group was reduced notably relative to control group, and the difference was remarkable $(P<0.05)$.

3.7. Contrast of IL-6 and IL-10 Levels in the Plasma of Two Groups of Patients Undergoing Laparoscopic Surgery. The levels of IL- 6 and IL-10 in the plasma of the two groups of patients were compared before the induction and after the operation. Figure 8(a) showed that IL-6 levels of control group and experimental group were greatly increased versus those at T1 $(P<0.05)$. IL-6 level of experimental group was notably reduced relative to that at $\mathrm{T} 5$, and the difference was very great $(P<0.05)$. Figure $8(\mathrm{~b})$ showed that IL-10 levels of control group and experimental group increased remarkably in contrast to those at $\mathrm{T} 1$, with marked differences $(P<0.05)$. IL-10 level of experimental group increased substantially versus that at T5 $(P<0.05)$.

3.8. Adverse Events in Patients Undergoing Laparoscopic Surgery. The two groups of patients were compared regarding the probability of related complications after the laparoscopy. There was no hypercapnia in experimental group and control group. $41 \%$ of patients were with 


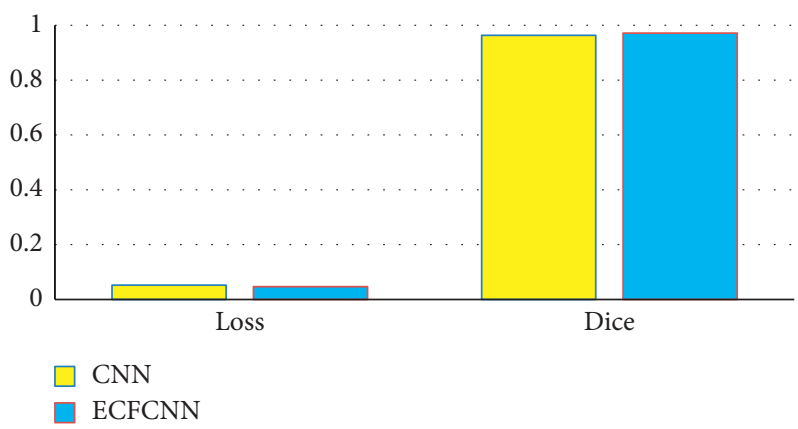

FIGURE 2: Simulation effect analysis of the two algorithms.

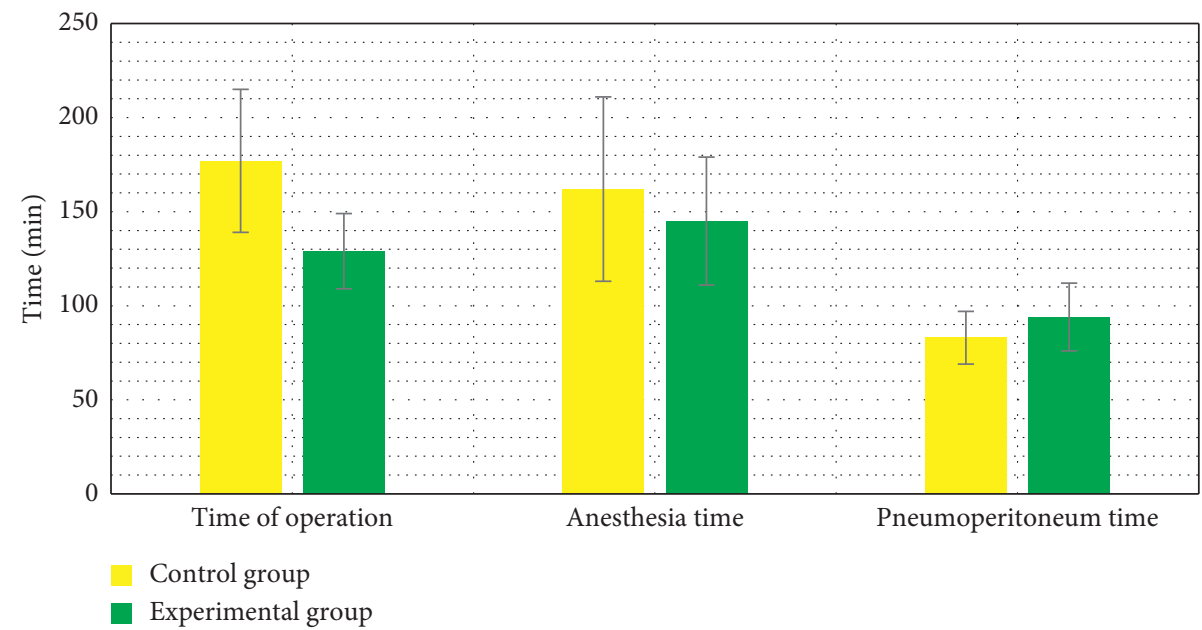

FIGURE 3: Contrast of operation time, anesthesia time, and pneumoperitoneum time between the two groups.

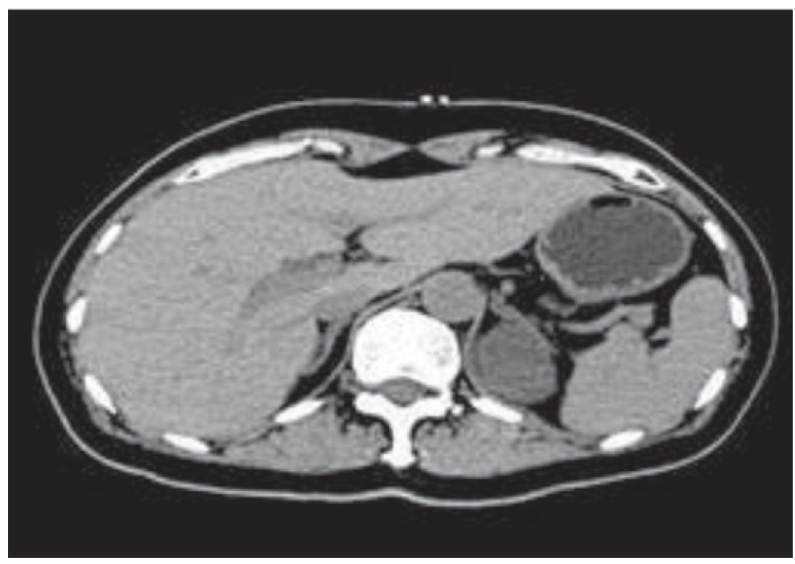

(a)

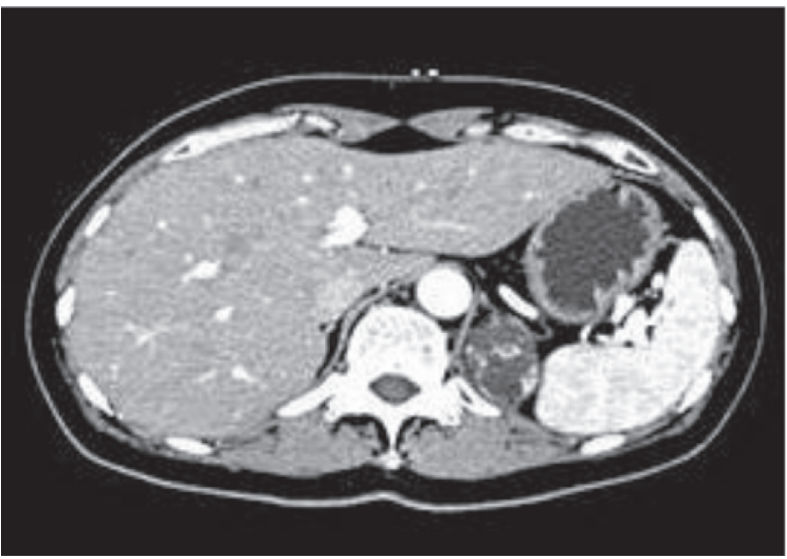

(b)

FIGURE 4: Continued. 


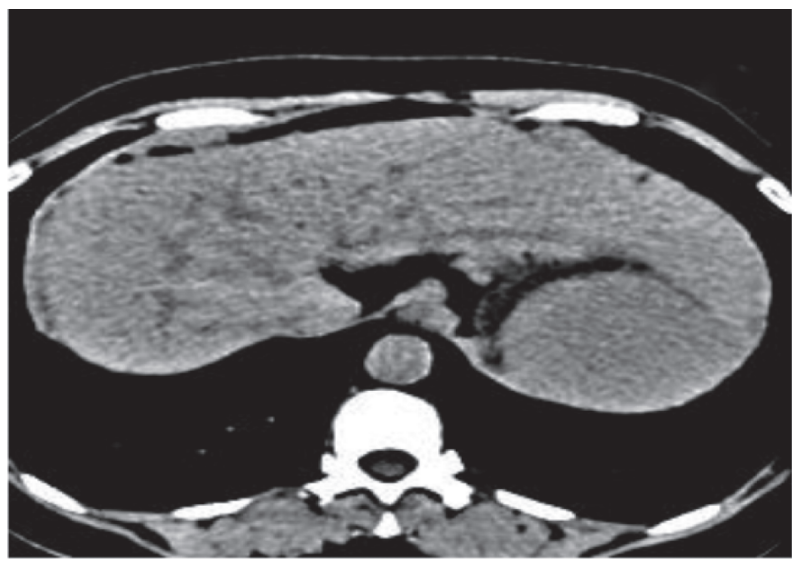

(c)

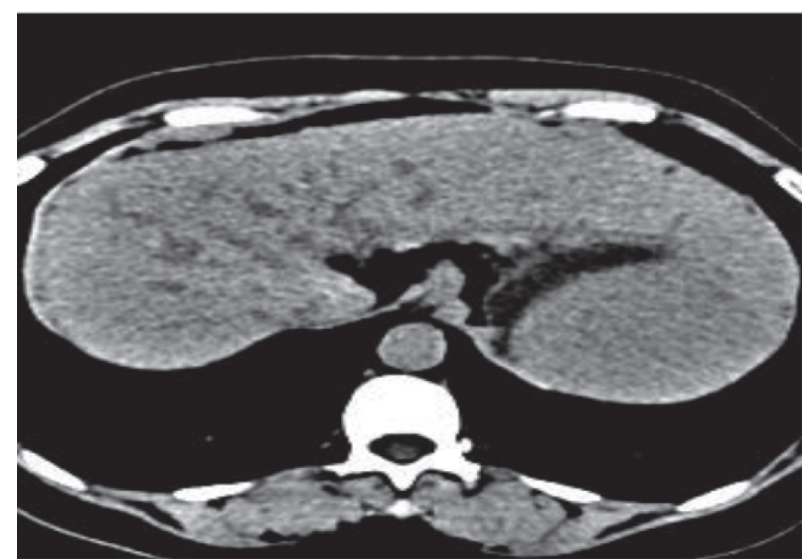

(d)

FIgURE 4: CT imaging characteristics. (a) The CT image of a 63-year-old female patient undergoing laparoscopic surgery to remove a large adrenal tumor. (b) The CT image of a 55-year-old male patient undergoing laparoscopic surgery to remove a large adrenal tumor. (c) and (d) The CT image of a 69-year-old male with liver cancer who underwent laparoscopic cholecystectomy.

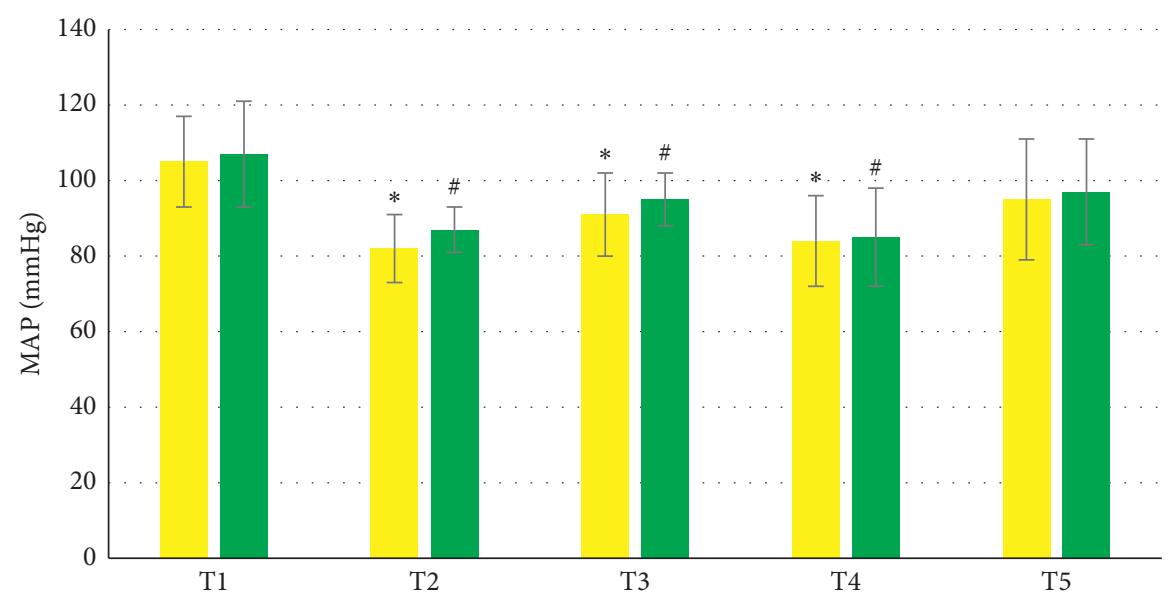

Control group

Experimental group

(a)

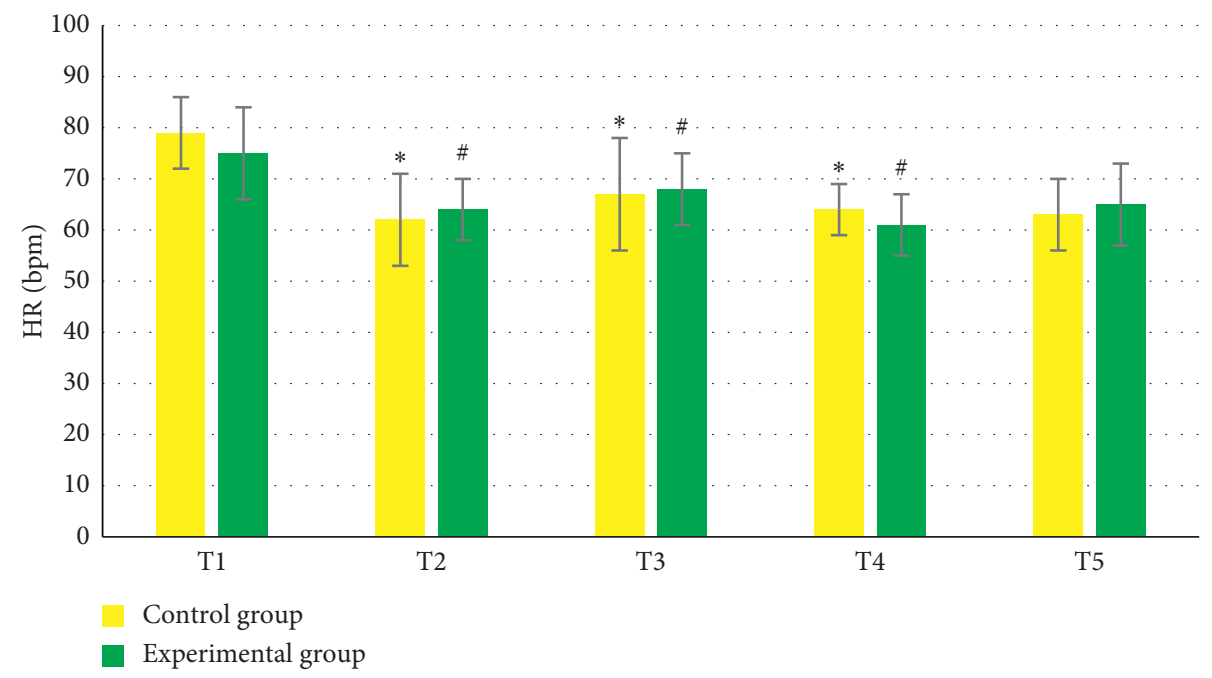

(b)

Figure 5: The dynamic changes of MAP and HR in the two groups. (a) The dynamic changes of MAP in the two groups. (b) The dynamic changes of HR in the two groups. Note. ${ }^{*}$ meant that, versus that at T1 in control group, the difference was substantial, $P<0.05$, while \# represented that, in contrast to that at $\mathrm{T} 1$ in experimental group, the difference was considerable, $P<0.05$. 


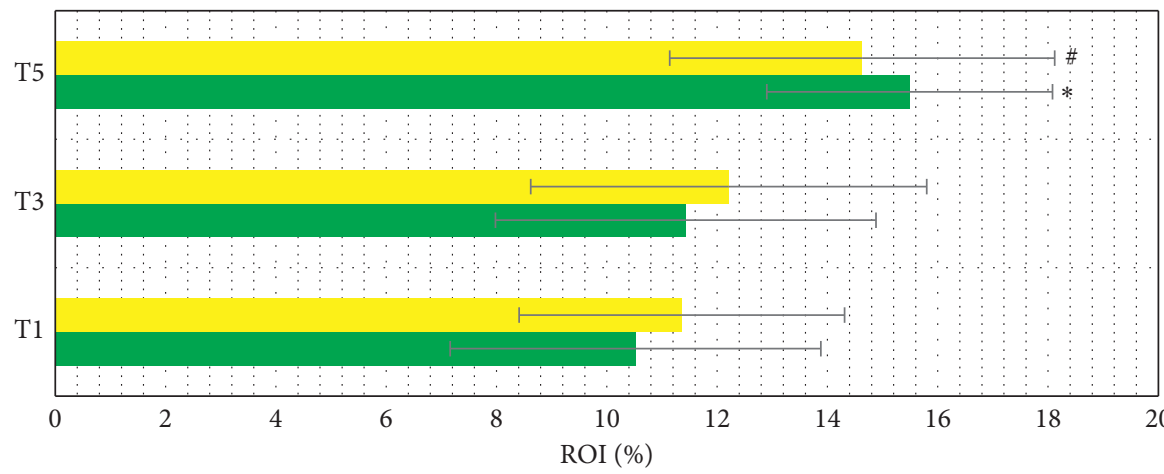

Experimental group

Control group

Figure 6: EIT monitoring data of two groups of patients. Note. ${ }^{*}$ meant that, versus that at T1 in control group, the difference was substantial, $P<0.05$, while \# represented that, in contrast to that at $\mathrm{T} 1$ in experimental group, the difference was considerable, $P<0.05$.

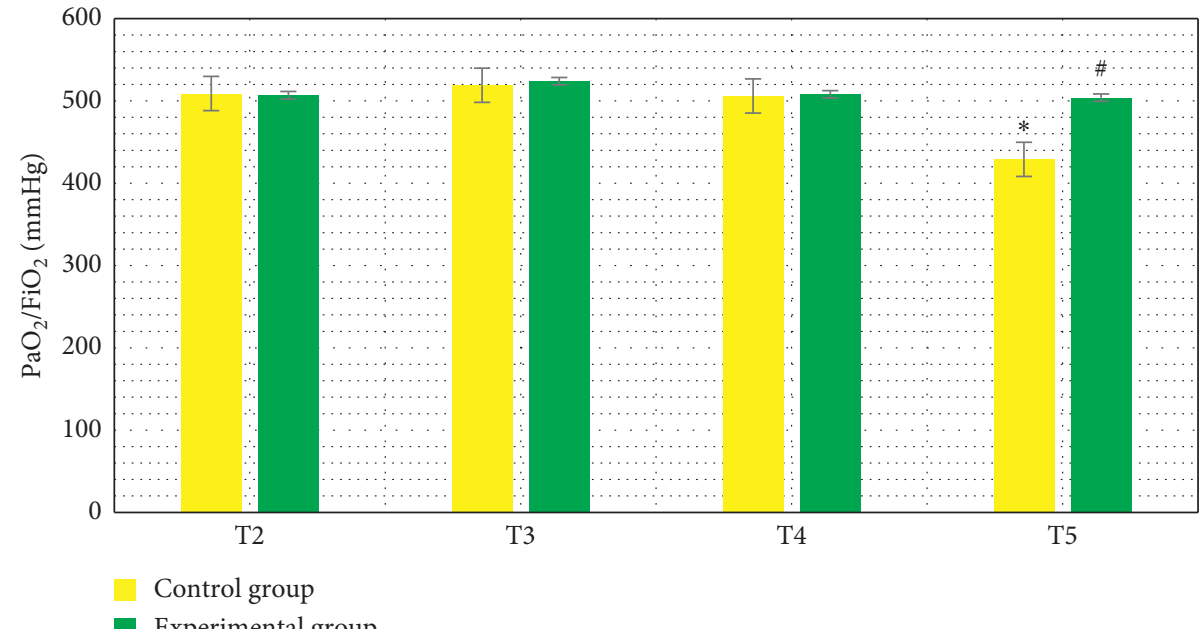

(a)

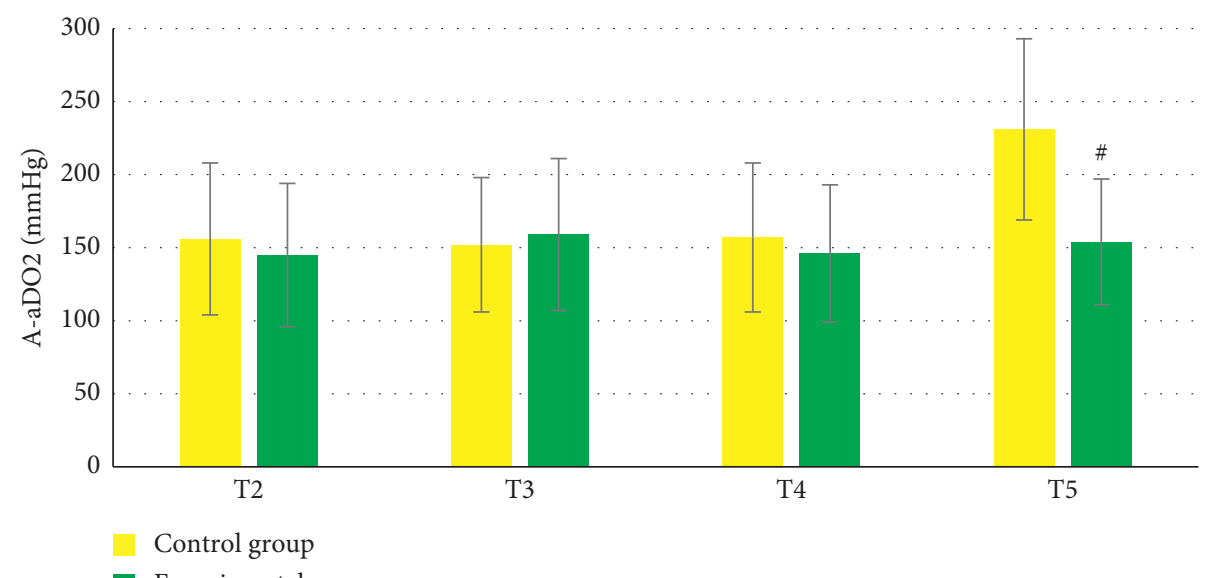

(b)

Figure 7: Contrast of oxygenation index $\left(\mathrm{PaO}_{2} / \mathrm{FiO}_{2}\right)$ and $\mathrm{A}-\mathrm{aDO}_{2}$ between the two groups. (a) Contrast of $\mathrm{PaO}_{2} / \mathrm{FiO}_{2}$ between the two groups. (b) Contrast of $\mathrm{A}-\mathrm{aDO}_{2}$ between the two groups. Note. ${ }^{*}$ meant that, versus that at $\mathrm{T} 2$ in control group, the difference was considerable, $P<0.05$, while ${ }^{\#}$ suggested that, in contrast to that at T5 in experimental group, the difference was substantial, $P<0.05$. 


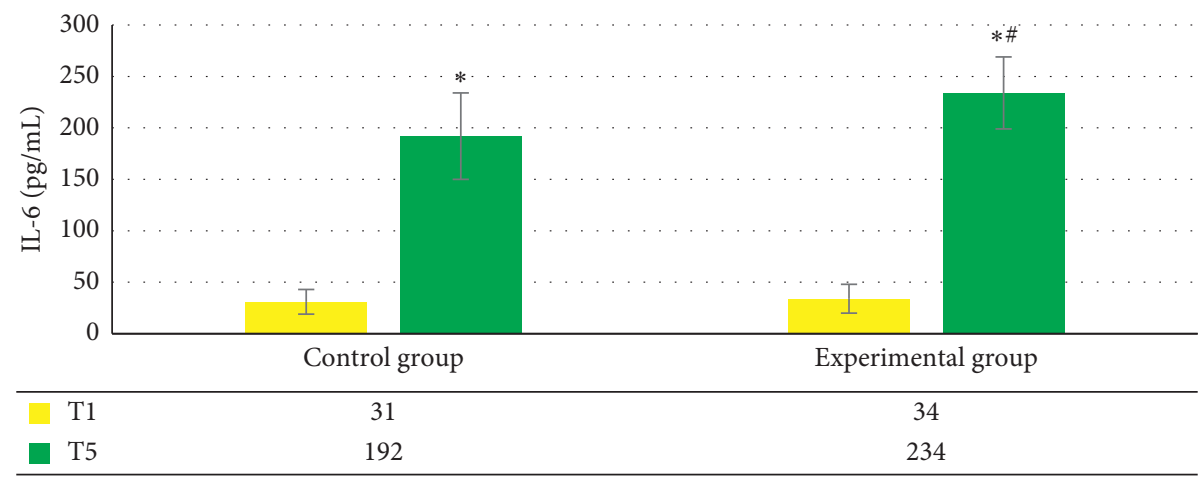

(a)

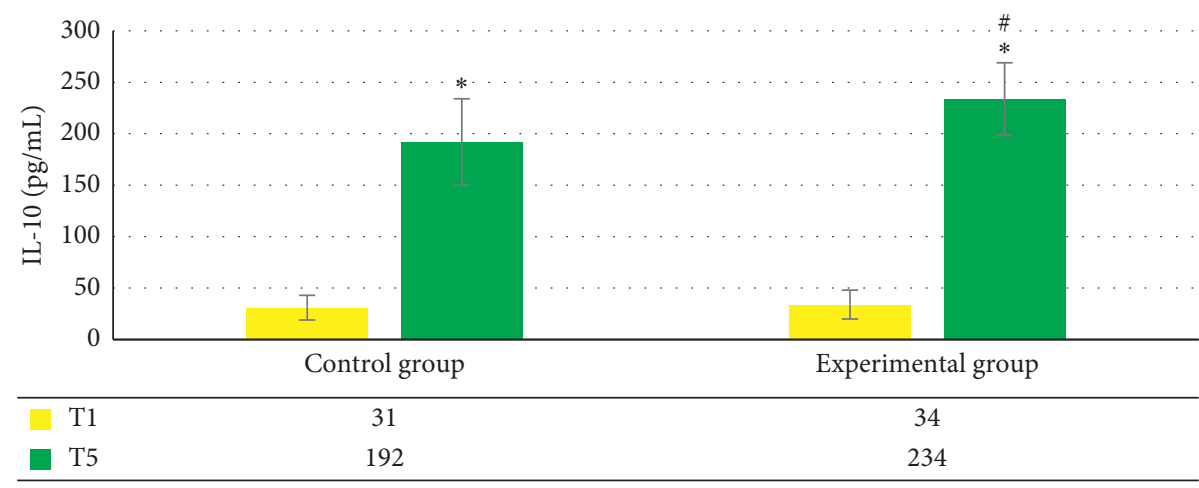

(b)

Figure 8: Contrast of IL-6 levels and IL-10 levels in the plasma of the two groups of patients. (a) Contrast of IL-6 levels in the plasma of the two groups of patients. (b) Contrast of IL-10 levels in the plasma of the two groups of patients. Note. ${ }^{*}$ meant that, in contrast to that at T1 in control group T1, $P<0.05$, while \# indicated that, relative to that at T5 in control group, $P<0.05$.

hypoxemia in control group and $32 \%$ in experimental group were with hypoxemia, showing no evident difference $(P>0.05)$. Postoperative restlessness occurred in $25 \%$ of control group and $13 \%$ of experimental group; similarly, no considerable difference was found $(P>0.05)$.

\section{Discussion}

The rapid development and related research of electrical impedance tomography (EIT) have promoted its wide clinical application. Using EIT monitoring, patients' spontaneous breathing and mechanical ventilation can be monitored dynamically in real time, and the respiratory function of patients can be evaluated. Laparoscopic surgery in different parts of the human body has different effects on the patient's respiratory function [9]. When surgery is performed in the lateral position, due to the combined effect of homeopathic lung pressure and abdominal ascending pressure, if the diaphragm muscle rises, it will cause atelectasis, which will prevent the patient from normal lung gas exchange. Carbon dioxide released from the blood cannot be normally eliminated from the body, and it is easy to accumulate carbon dioxide acidosis. If diagnosis and treatment are not performed in time, it will directly affect the patient's respiratory function [10].

Full convolutional neural network was first proposed for image semantic segmentation and then gradually applied to image classification, target detection, and other image processing tasks. Compared with convolutional neural network, full convolutional neural network has smaller parameters and calculations and can process input images with different resolutions, which is more efficient and flexible. In this study, based on deep learning full convolutional neural network and cavity convolution, a full convolutional neural network optimization (ECFCNN) algorithm was proposed for lung CT image segmentation. The results showed that the loss of CNN model was 0.0523 and the Dice coefficient was 0.9635 . The loss of ECFCNN model was 0.0469 , and the Dice coefficient was 0.9712 . The loss function of ECFCNN model was slightly better than that of $\mathrm{CNN}$ model and Dice coefficient.

The main indexes of the patient's pulmonary function include oxygenation index $\left(\mathrm{PaO}_{2} / \mathrm{FiO}_{2}\right), \mathrm{A}-\mathrm{aDO}_{2}$, and RI. The oxygenation index can reflect the oxygenation of the lungs. $\mathrm{A}-\mathrm{aDO}_{2}$ can reflect the oxygen diffusion capacity of the lungs, that is, the ability of the alveoli to perform ventilation. When its value gradually increases, the diffusion function of the lungs is abnormal, which is one of the important indexes to evaluate the damage of the pulmonary function [11]. RI is an important index reflecting the oxygen exchange function of the lungs. When it is greater than 1 , the oxygen exchange function is significantly reduced [12]. Gray et al. [13] studied the effect of positive end-expiratory pressure during laparoscopic surgery on improving the lung 
oxygenation function of patients. The results showed that the oxygenation index increased, and the $\mathrm{A}-\mathrm{aDO}_{2}$ and the RI decreased, indicating that such mode can improve the lung ventilation function and was beneficial to the lung gas exchange. In this work, the lung oxygenation index of control group at T2 and T5 decreased obviously, indicating that the lungs were mechanically damaged due to excessive expansion of the alveoli during pneumoperitoneum. Compared to that at $\mathrm{T} 1$, the $\mathrm{HR}$ of $\mathrm{T} 2-\mathrm{T} 5$ was greatly reduced, but there was no significant difference between MAP and $\mathrm{SpO}_{2}$, indicating that laparoscopic surgery had no obvious effect on the patient's hemodynamic parameters. The results of this study were similar to those of Kim et al. [14], who assessed the influence of laparoscopic surgery on hemodynamics. It was revealed that no notable difference was found in hemodynamics and pneumoperitoneum between experimental group and control group.

Inflammatory cells are very easy to accumulate in the lungs. Once acute lung injury occurs, the inflammatory cells will be activated in a short time and release massive inflammatory factors [15]. Zhang and Liang [16] pointed out that activating inflammatory cells at the cellular level would cause a series of inflammatory chain reactions, releasing a large number of inflammatory mediators and causing lung damage. The levels of inflammatory factors IL- 6 and IL-10 in plasma were determined at $\mathrm{T} 1$ and $\mathrm{T} 5$ time points, and the effects of laparoscopic surgery on the levels of inflammatory factors were explored. The levels of IL- 6 and IL-10 in control group and experimental group both increased notably. The IL-6 level of experimental group was greatly inferior to control group at T5. Therefore, it was verified that laparoscopic surgery for pneumoperitoneum can effectively reduce the inflammation of the lungs, which was consistent with the above research reports.

\section{Conclusion}

Based on deep learning, ECFCNN algorithm was proposed, which was compared with $\mathrm{CNN}$ algorithm and applied to the evaluation of pulmonary function of 60 patients undergoing laparoscopic surgery. Not only can the segmented images of the ECFCNN model describe the overall outline of the lung, but also the definition of the edge outline was higher than that of the CNN model. EIT-guided lung protection ventilation can help assess the pulmonary function of patients undergoing laparoscopic surgery, significantly reduce the incidence of atelectasis, and improve lung oxygenation during and after surgery. The shortcoming of this work is that the blood collection time of patients after surgery is not prolonged, and the changes in the level of inflammatory factors cannot be assessed. In short, this work has well confirmed that CT images guided by EIT can monitor the clinical effects of lung protective ventilation in real time and provide a reference diagnostic basis for clinical laparoscopic surgery.

\section{Data Availability}

The data used to support the findings of this study are available from the corresponding author upon request.

\section{Conflicts of Interest}

The authors declare no conflicts of interest.

\section{References}

[1] I. Frerichs, M. B. P. Amato, A. H. van Kaam et al., "Chest electrical impedance tomography examination, data analysis, terminology, clinical use and recommendations: consensus statement of the translational EIT development study group," Thorax, vol. 72, no. 1, pp. 83-93, 2017.

[2] C. Nestler, P. Simon, D. Petroff et al., "Individualized positive end-expiratory pressure in obese patients during general anaesthesia: a randomized controlled clinical trial using electrical impedance tomography," British Journal of Anaesthesia, vol. 119, no. 6, pp. 1194-1205, 2017.

[3] S. Białka, M. Copik, K. Rybczyk, and H. Misiołek, "Electrical impedance tomography for diagnosis and monitoring of pulmonary function disorders in the intensive care unit - case report and review of literature," Anaesthesiology Intensive Therapy, vol. 49, no. 3, pp. 222-226, 2017.

[4] Z. Lv, X. Li, H. Lv, and W. Xiv, "BIM big data storage in WebVRGIS," IEEE Transactions on Industrial Informatics, vol. 16, no. 4, pp. 2566-2573, 2019.

[5] D. Meroni, C. C. Maglioli, D. Bovio, F. G. Greco, and A. Aliverti, "An electrical impedance tomography (EIT) multi-electrode needle-probe device for local assessment of heterogeneous tissue impeditivity," Annual International Conference of the IEEE Engineering in Medicine and Biology Society. IEEE Engineering in Medicine and Biology Society. Annual International Conference, vol. 2017, pp. 1385-1388, 2017.

[6] T. Akiyoshi, "Technical feasibility of laparoscopic extended surgery beyond total mesorectal excision for primary or recurrent rectal cancer," World Journal of Gastroenterology, vol. 22, no. 2, pp. 718-726, 2016.

[7] Y. Chen, S. Hu, H. Mao, W. Deng, and X. Gao, "Application of the best evacuation model of deep learning in the design of public structures," Image and Vision Computing, vol. 102, Article ID 103975, 2020.

[8] E. J. White, M. McMahon, M. T. Walsh, J. C. Coffey, and L. O'Sullivan, "Toward a model of human information processing for decision-making and skill acquisition in laparoscopic colorectal surgery," Journal of Surgical Education, vol. 75, no. 3, pp. 749-757, 2018.

[9] S. Han, J. Yang, and J. Xu, "Deep learning-based computed tomography image features in the detection and diagnosis of perianal abscess tissue," Journal of Healthcare Engineering, vol. 2021, Article ID 3706265, 2021.

[10] S. A. Antoniou, S. Morales-Conde, S. Morales-Conde et al., "Single-incision laparoscopic surgery through the umbilicus is associated with a higher incidence of trocar-site hernia than conventional laparoscopy: a meta-analysis of randomized controlled trials," Hernia, vol. 20, no. 1, pp. 1-10, 2016.

[11] L. Han, Y. Chen, W. Cheng, H. Bai, J. Wang, and M. Yu, "Deep learning-based CT image characteristics and postoperative anal function restoration for patients with complex anal fistula," Journal of Healthcare Engineering, vol. 2021, Article ID 1730158, 2021.

[12] C. Guo, G. Hoek, L. Y. Chang et al., "Long-term exposure to ambient fine particulate matter (PM2.5) and lung function in children, adolescents, and young adults: a longitudinal cohort study," Environmental Health Perspectives, vol. 127, no. 12, Article ID 127008, 2019. 
[13] D. M. Gray, L. Turkovic, L. Willemse et al., "Lung function in african infants in the drakenstein child health study. impact of lower respiratory tract illness," American Journal of Respiratory and Critical Care Medicine, vol. 195, no. 2, pp. 212-220, 2017.

[14] S. K. Kim, J. C. Bae, J. H. Baek et al., "Decline in lung function rather than baseline lung function is associated with the development of metabolic syndrome: a six-year longitudinal study," PloS One, vol. 12, no. 3, Article ID e0174228, 2017.

[15] C. Leiva-Salinas, B. Jiang, and M. Wintermark, "Computed tomography, computed tomography angiography, and perfusion computed tomography evaluation of acute ischemic stroke," Neuroimaging Clinics of North America, vol. 28, no. 4, pp. 565-572, 2018.

[16] X. H. Zhang and H. M. Liang, "Systematic review with network meta-analysis: diagnostic values of ultrasonography, computed tomography, and magnetic resonance imaging in patients with ischemic stroke," Medicine, vol. 98, no. 30, Article ID e16360, 2019. 